\title{
G-Numbers: Importance-Necessity Concept in Uncertain Environment
}

\author{
Saeid Jafarzadeh Ghoushchi*, Mohammad Khazaeili \\ Faculty of Industrial Engineering, Urmia University of Technology, Urmia, Iran \\ Email address: \\ s.jafarzadeh@uut.ac.ir (S. J. Ghoushchi), saeid.edu@gmail.com (S. J. Ghoushchi) \\ ${ }^{*}$ Corresponding author
}

To cite this article:

Saeid Jafarzadeh Ghoushchi, Mohammad Khazaeili. G-Numbers: Importance-Necessity Concept in Uncertain Environment. International Journal of Management and Fuzzy Systems. Vol. 5, No. 1, 2019, pp. 27-32. doi: 10.11648/j.ijmfs.20190501.15

Received: March 20,2019; Accepted: May 6, 2019; Published: May 30, 2019

\begin{abstract}
Decisions are mainly grounded on information; therefore, the information should have the least ambiguity and uncertainty to make beneficial and reliable decisions. Many concepts such as fuzzy sets theory, Z-Numbers, and D-Numbers, have been proposed. All the previous concepts have some desirable properties while they do not consider the concept of necessity. In this paper, a new concept, named as G-numbers is proposed to reduce the uncertainty of information based on importance and necessity concepts. In a G-numbers, $\mathrm{G}=(\mathrm{I}, \mathrm{N})$, I is the Importance component and $\mathrm{N}$ is the Necessity component on the real-valued uncertain variables. In general, I and $\mathrm{N}$ are described as linguistic variables, Examples: an appointment (high, very high); investment in the stock market (high, medium). An ordered pair relates to computations with Gnumbers. In this study, the concept of a G-number is introduced, and the arithmetic operations on G-numbers are presented. Finally, a numerical example is used to illustrate the efficiency of the proposed approach. The concept of G-numbers can be used for a wide range of practical issues in various areas, such as inter alia, social, economic, and risk assessment, and decision-making.
\end{abstract}

Keywords: Importance, Necessity, Uncertain Information, Fuzzy Numbers, G-Numbers, Decision Making

\section{Introduction}

In the real world, a considerable proportion of information in planning and decision-making issues is accompanied by uncertainty. Humans can make rational decisions based on inaccurate or incomplete information; accordingly, achieving organizational objectives is a crucial challenge. To date, several concepts and theories have been proposed. Zadeh in 1965 [1] presented fuzzy set theory to reduce uncertainty due to ambiguity in information. Zadeh in 2011 [2] also introduced the Z-Numbers concept by considering the uncertainty along with the reliability component. Yong Deng in 2012 [3], proposed a new concept, called D numbers, to be more effective in representing various types of uncertainty. D numbers is an extension of the Dempster-Shafer theory. Recently, R-Number was presented by seiti et al in 2019 [4] in order to reduce the risk of information. These concepts have been applied in many fields such as supplier selection [4-7], risk assessment [4, 8-10], information management [4,

\section{$11,12]$, etc.}

One of the key indicators of decision-making that has been considered so far is the concept of importance. The importance of an activity or a measure refers to its value and importance to the environment and prevailing conditions. However, sometimes there is no or excessively less necessity to implement the activities and measures that seem to be importance. It should also be notified that there are sometimes several importance and potential issues in a process, but only a few special ones are necessary to be taken into account. Hitherto, importance and necessity in decisionmaking problems have been applied interchangeably as a single concept. Thus, necessity has been neglected as an independent indicator. Integrating importance and necessity as two independent concepts can reduce uncertainty in decision-making and planning. Indeed, taking into account the importance and necessity concepts in decisions issues can lead to the following results:

Enabling a concurrent awareness of the importance and necessity of activity or criteria (raising awareness). 
Making more reasonable arguments to decide on an issue (reducing uncertainty).

Making managers and decision makers more informed of and sensitive to choices and prioritizations (reducing ambiguity).

Justifying the necessity, in addition to the importance, of carrying out some activities in light of the prevailing circumstances and time (reducing the consequences resulting from the lack of timely implementation of some activities).

In this study, the concept of G-information is combined with multiple criteria decision-making (MCDM) methods, and a framework is proposed. The main purpose of the integration at issue is to enhance the decision maker's ability to reduce ambiguity in the decision-making process. Indeed, this integration provides the ability to create a managerial structure among the components of the decision-making matrix.

In the next section, a brief introduction of the background knowledge is represented. Then, an overview of G-numbers is provided. Subsequently, an illustrative numerical example is presented, and finally, the conclusions are drawn.

\section{Background Knowledge}

For the first time in 1954, the then president of the United States of America, Eisenhower, offered Urgent-Important Principle for planning and decision-making during his international speech to the Second Assembly of the World Council of Churches [13, 14].

At that time, Eisenhower noticed that proper management of activities refers to not only performing all tasks, but also efficiently performing the tasks. In other words, prioritizing important tasks regardless of their extent of necessity and urgency may lead to the reduction of effectiveness and efficiency of management. Accordingly, Eisenhower suggested a matrix, aka Urgent-Important Matrix, which is based on the concepts of important and urgency. This matrix included four quadrants: important and urgent (leads to DO); important, but not urgent (leads to SCHEDULE); urgent, but not important (leads to DELEGATE); and neither important nor urgent (leads to ELIMINATE). He suggested to classifying the list of tasks under each of these categories on the basis of the concepts of important and urgency and implementing them as the desired management strategy (Figure 1).

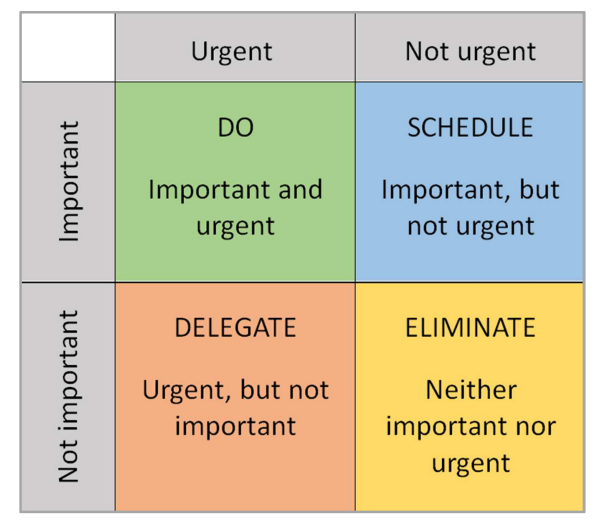

Figure 1. Eisenhower matrix [14].
Given the existing resources and conditions of an organization, importance-based planning may lead to the achievement of the organizational macro-level goals, and urgency-based planning may reduce the consequences of failure to timely handle a series of activities. Hence, simultaneous consideration of the two concepts of importance and urgency seems essential to achieve high efficiency and profitability in organizations.

Researchers generally consider the concept of importance in MCDM issues [15, 16], while taking the concept of necessity, which can be a comprehensive equivalent for urgency, into account can reduce ambiguity and get decisionmaking closer to the real-world condition. It should be noted that in many decision-making problems, the concepts can be expressed as G-numbers.

The ordered triple $(X, I, N)$ is referred to as a G-valuation. $\mathrm{X}$ is an unspecified variable, $\mathrm{I}$ and $\mathrm{N}$ are Importance and Necessity component, respectively, which can take the value of X. For example, (Increase in the educational budget, Medium, High) is referred to as a G-valuation. A collection of $\mathrm{G}$-valuations is referred to as G-information. In many decisionmaking issues, concepts can be expressed in the form of Ginformation. Additional examples are provided as follows:

A Board Meeting: Importance (Low) and Necessity (High). Vacation Travel: Importance (Low) and Necessity (Very Low).

Installing Fire Protection Systems: Importance (Very high) and Necessity (High).

Buying a New Home: Importance (High) and Necessity (Low).

In this research, the linguistic variables such as triangular fuzzy numbers are used for the concepts of importance and necessity, and a new approach is proposed to enhance the decision maker's ability to reduce ambiguity in decision issues. The results can illustrate the impact of taking into account the concept of necessity in the decision-making process. Notably, G-numbers can be a combination of various planning and decision-making issues.

\section{G-Numbers: A New Methodology}

In this section, the basic concepts utilized in this study are explained. Some definitions of G-numbers are proposed for analysis, and the arithmetic operations on these G-numbers are defined. Definition 1 [17]: A fuzzy number $\tilde{A}=(l, m, u)$ is said to be a triangular fuzzy number (figure 2) where $l, m$ and $u$ respectively represent the lower, modal, and upper value. The membership function of the triangular fuzzy number is defined by:

$$
\mu_{\tilde{A}}(x)=\left\{\begin{array}{lc}
\frac{x-l}{m-l}, & l \leq x \leq m \\
1, & m \leq x \leq u \\
\frac{u-x}{u-m}, & \text { otherwise }
\end{array}\right.
$$




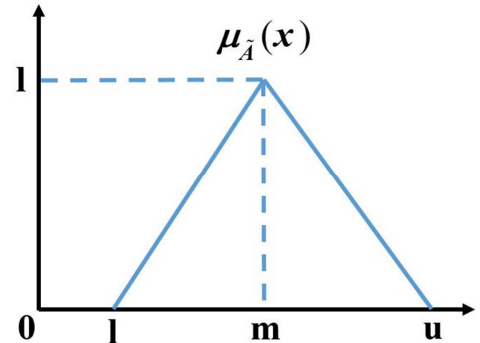

Figure 2. A triangular fuzzy number.

Definition 2[17]: A triangular fuzzy number $(l, m, u)$ is said to be non-negative if $l \geq 0$.

Definition 3: A G-Numbers can be defined as $G=(\tilde{I}, \tilde{N})$, where $\tilde{I}=\left(l_{I}, m_{I}, u_{I}\right)$ is the Importance component and $\tilde{N}=\left(l_{N}, m_{N}, u_{N}\right)$ is the Necessity component, which are triangular fuzzy numbers.

Definition 4: A G-Numbers $\left[\left(l_{I}, m_{I}, u_{I}\right),\left(l_{N}, m_{N}, u_{N}\right)\right]$ is said to be non-negative if $l_{I} \geq 0, l_{N} \geq 0$.

Definition 5: Let $G_{1}=\left[\left(l_{I}^{1}, m_{I}^{1}, u_{I}^{1}\right),\left(l_{N}^{1}, m_{N}^{1}, u_{N}^{1}\right)\right]$ and $G_{2}=\left[\left(l_{I}^{2}, m_{I}^{2}, u_{I}^{2}\right),\left(l_{N}^{2}, m_{N}^{2}, u_{N}^{2}\right)\right]$ be two non-negative GNumbers and $\lambda$ be the non-negative variable of real numbers. The arithmetic operations are defined as follows.

$$
\begin{aligned}
\text { i) } G_{1} \oplus G_{2} & =\left[\left(l_{I}^{1}+l_{I}^{2}, m_{I}^{1}+m_{I}^{2}, u_{I}^{1}+u_{I}^{2}\right),\left(l_{N}^{1}+l_{N}^{2}, m_{N}^{1}+m_{N}^{2}, u_{N}^{1}+u_{N}^{2}\right)\right] \\
\text { ii) } G_{1} \ominus G_{2} & =\left[\left(l_{I}^{1}-u_{I}^{2}, m_{I}^{1}-m_{I}^{2}, u_{I}^{1}-l_{I}^{2}\right),\left(l_{N}^{1}-u_{N}^{2}, m_{N}^{1}-m_{N}^{2}, u_{N}^{1}-l_{N}^{2}\right)\right] \\
\text { iii) } G_{1} \otimes G_{2} & =\left[\left(l_{I}^{1} l_{I}^{2}, m_{I}^{1} m_{I}^{2}, u_{I}^{1} u_{I}^{2}\right),\left(l_{N}^{1} l_{N}^{2}, m_{N}^{1} m_{N}^{2}, u_{N}^{1} u_{N}^{2}\right)\right] \\
\text { iv) } G_{1} \oslash G_{2} & =\left[\left(l_{I}^{1} / u_{I}^{2}, m_{I}^{1} / m_{I}^{2}, u_{I}^{1} / l_{I}^{2}\right),\left(l_{N}^{1} / u_{N}^{2}, m_{N}^{1} / m_{N}^{2}, u_{N}^{1} / l_{N}^{2}\right)\right] \\
\text { v) } \lambda G_{1} & =\left[\left(\lambda l_{I}^{1}, \lambda m_{I}^{1}, \lambda u_{I}^{1}\right),\left(\lambda l_{N}^{1}, \lambda m_{N}^{1}, \lambda u_{N}^{1}\right)\right]
\end{aligned}
$$

Definition 6: Let $(\tilde{I}, \tilde{N})$ be a G-Number. The transformation rules for $\mathrm{G}$ - numbers linguistic variables is defined as:

$$
G=\alpha \tilde{I} \oplus \beta \tilde{N} ; \quad \alpha+\beta=1 ; \alpha, \beta \geq 0
$$

$\alpha$ and $\beta$ represent the non-negative weight of the Importance and Necessity component, respectively.

\section{Application}

The G-Numbers concept can be applied in various planning and decision-making issues. In this section, the combination of G-numbers with a decision making problem is presented. To solve decision making problems, first, the decision matrix is expressed in a matrix format as:

$$
\begin{aligned}
& \begin{array}{llll}
C_{1} & C_{2} & \ldots & C_{n}
\end{array} \\
& \begin{array}{c}
A_{1} \\
A_{2} \\
\vdots \\
A_{m}
\end{array}\left[\begin{array}{cccc}
G_{11} & G_{12} & \cdots & G_{1 n} \\
G_{21} & G_{22} & \cdots & G_{2 n} \\
\vdots & \vdots & \ddots & \vdots \\
G_{m 1} & G_{m 2} & \cdots & G_{m n}
\end{array}\right]
\end{aligned}
$$

Where $G_{i j}, \forall i, j$ and $w_{j}, j=1,2, \ldots, n$ are G-Numbers. The following G-matrix is assumed to show the score $\left(G_{11}, G_{12}, \ldots, G_{m n}\right)$ received by alternatives $\left(A_{1}, A_{2}, \ldots, A_{m}\right)$ with respect to the criteria $\left(C_{1}, C_{2}, \ldots, C_{n}\right)$. Thus, $G_{i j}=\left(\tilde{I}_{i j}, \tilde{N}_{i j}\right)$, where $\tilde{I}_{i j}$ and $\tilde{N}_{i j}$ are the Importance and
Necessity components of ith $(i=1, \ldots, m)$ alternative to jth $(j=1, \ldots, n)$ criteria. And $w_{j}=\left(\tilde{w}_{j}^{I}, \tilde{w}_{j}^{N}\right)$ where $\tilde{w}_{j}^{I}$ and $\tilde{w}_{j}^{N}$ are respectively the importance and necessity weights of criteria. And the component of $G_{i j}$ and $w_{j}$ are linguistics variables that can be described by triangular fuzzy numbers.

By using Definition 6, the components of the evaluation G-matrix and weights of criteria, which are transformed into a fuzzy evaluation matrix, are defined as follows:

$$
\begin{aligned}
& G_{i j}=\alpha \tilde{I}_{i j} \oplus \beta \tilde{N}_{i j} \quad, \quad W_{j}=\alpha \tilde{w}_{j}^{I} \oplus \beta \tilde{w}_{j}^{N} \\
& \alpha+\beta=1 \quad ; \alpha, \beta \geq 0
\end{aligned}
$$

In the following, the linguistics variables of G-Numbers for the Ratings and weights of various criteria can be expressed in triangular fuzzy numbers as Table 1 and 2.

Table 1. Linguistics variables of G-Numbers for the alternative ratings.

\begin{tabular}{lll}
\hline L.V. & Importance & Necessity \\
\hline Very poor (VP) & $(0,0,1)$ & $(0,0,1.5)$ \\
Poor (P) & $(0,1,3)$ & $(0,1.5,3)$ \\
Medium poor (MP) & $(1,3,5)$ & $(1.5,3,4.5)$ \\
Fair (F) & $(3,5,7)$ & $(3,4.5,6)$ \\
Medium good (MG) & $(5,7,9)$ & $(4.5,6,7.5)$ \\
Good (G) & $(7,9,10)$ & $(6,7.5,9)$ \\
Very good (VG) & $(9,10,10)$ & $(7.5,9,10)$ \\
\hline
\end{tabular}


Table 2. Linguistics variables of G-Numbers for the weight of each criteria.

\begin{tabular}{lll}
\hline L.V. & Importance & Necessity \\
\hline Very low (VL) & $(0,0,0.1)$ & $(0,0,0.15)$ \\
Low (L) & $(0,0.1,0.3)$ & $(0,0.15,0.3)$ \\
Medium low (ML) & $(0.1,0.3,0.5)$ & $(0.15,0.3,0.45)$ \\
Medium (M) & $(0.3,0.5,0.7)$ & $(0.3,0.45,0.6)$ \\
Medium high (MH) & $(0.5,0.7,0.9)$ & $(0.45,0.6,0.75)$ \\
High (H) & $(0.7,0.9,1.0)$ & $(0.6,0.75,0.9)$ \\
Very high (VH) & $(0.9,1.0,1.0)$ & $(0.75,0.9,1.0)$ \\
\hline
\end{tabular}

\section{Numerical Example}

In this section, a supplier selection problem is considered. Six suppliers $\left(A_{1}, A_{2}, \ldots, A_{6}\right)$ with four criteria, namely, $\mathrm{C}_{1}$ (cost), $\mathrm{C}_{2}$ (quality), $\mathrm{C}_{3}$ (delivery time), and $\mathrm{C}_{4}$ (Environmental
Management System), are considered. Data associated with attention to the supplier's specifications by the decision maker, such as whether domestic or foreign, whether branded or non-branded, country of origin, type of product, and production time, are presented in Table 3.

Table 3. Evaluation Information by rating for each alternatives in form of G-numbers.

\begin{tabular}{lllll}
\hline & $\mathbf{C}_{1}$ & $\mathbf{C}_{2}$ & $\mathbf{C}_{3}$ & $\mathbf{C}_{4}$ \\
\hline $\mathrm{A}_{1}$ & $(\mathrm{G}, \mathrm{VG})$ & $(\mathrm{VG}, \mathrm{MP})$ & $(\mathrm{P}, \mathrm{G})$ & $(\mathrm{F}, \mathrm{MP})$ \\
$\mathrm{A}_{2}$ & $(\mathrm{~F}, \mathrm{G})$ & $(\mathrm{VG}, \mathrm{G})$ & $(\mathrm{P}, \mathrm{MG})$ & $(\mathrm{MP}, \mathrm{G})$ \\
$\mathrm{A}_{3}$ & $(\mathrm{G}, \mathrm{MG})$ & $(\mathrm{MP}, \mathrm{G})$ & $(\mathrm{MG}, \mathrm{MP})$ & $(\mathrm{MG}, \mathrm{F})$ \\
$\mathrm{A}_{4}$ & $(\mathrm{VG}, \mathrm{G})$ & $(\mathrm{P}, \mathrm{G})$ & $(\mathrm{MG}, \mathrm{VG})$ & $(\mathrm{MP}, \mathrm{MP})$ \\
$\mathrm{A}_{5}$ & $(\mathrm{G}, \mathrm{G})$ & $(\mathrm{F}, \mathrm{F})$ & $(\mathrm{G}, \mathrm{MG})$ & $(\mathrm{F}, \mathrm{F})$ \\
$\mathrm{A}_{6}$ & $(\mathrm{VG}, \mathrm{F})$ & $(\mathrm{G}, \mathrm{MG})$ & $(\mathrm{MG}, \mathrm{MP})$ & $(\mathrm{M}, \mathrm{ML})$ \\
Weight & $(\mathrm{H}, \mathrm{MH})$ & $(\mathrm{VH}, \mathrm{M})$ & $(\mathrm{MH}, \mathrm{H})$ & \\
\hline
\end{tabular}

In table 3, the weights of criteria and the evaluation matrix of G-Numbers (G-matrix) are considered by the decision maker. By using Definition 6, $\left(G_{i j}=\alpha I_{i j} \oplus \beta N_{i j} ; W_{j}=\alpha \tilde{w}_{j}^{I} \oplus \beta \tilde{w}_{j}^{N}\right)$, and with suppose $\alpha=0.7$ and $\beta=0.3$ (weights of importance and necessity, respectively), The G-Numbers evaluation matrix is converted to the fuzzy evaluation matrix.

For example, by using tables 1 and 2, $(G, V G),(H, M H)$

components are displayed in the form of $G_{11}=[(7,9,10) ;(7.5,9,10)], W_{C_{1}}=[(0.7,0.9,1.0) ;(0.45,0.6,0.75)]$. $G_{11}$ is converted as follows:

$$
\begin{aligned}
& G_{11}=0.7 *(7,9,10) \oplus 0.3 *(7.5,9,10)=(7.15,9,10) \\
& W_{C_{1}}=0.7 *(0.7,0.9,1.0) \oplus 0.3 *(0.45,0.6,0.75)=(0.625,0.81,0.925)
\end{aligned}
$$

All the members in Tables 3 are transformed, as presented in Table 4, by repeating the same procedure.

\begin{tabular}{|c|c|c|c|c|}
\hline & C1 & $\mathrm{C2}$ & C3 & $\mathrm{C4}$ \\
\hline A1 & $(7.15,9,10)$ & $(6.75,7.9,8.35)$ & $(1.8,2.95,4.8)$ & $(2.55,4.4,6.25)$ \\
\hline $\mathrm{A} 2$ & $(3.9,5.75,7.6)$ & $(8.1,9.25,9.7)$ & $(1.35,2.5,4.35)$ & $(2.5,4.35,6.2)$ \\
\hline A3 & $(6.25,8.1,9.25)$ & $(2.5,4.35,6.2)$ & $(6.75,7.9,8.35)$ & $(4.4,6.25,8.1)$ \\
\hline A4 & $(8.1,9.25,9.7)$ & $(1.8,2.95,4.8)$ & $(5.75,7.6,9.3)$ & $(6.25,8.1,9.25)$ \\
\hline A5 & $(6.7,8.55,9.7)$ & $(3,4.85,6.7)$ & $(6.25,8.1,9.25)$ & $(1.15,3,4.85)$ \\
\hline A6 & $(7.2,8.35,8.8)$ & $(6.25,8.1,9.25)$ & $(3.95,5.8,7.65)$ & $(3,4.85,6.7)$ \\
\hline
\end{tabular}

Table 4. Transformation rules for G-numbers linguistic variables to fuzzy numbers.

The ranking of suppliers by fuzzy TOPSIS method Chen [18] is shown in Table 4. The final ranking results are presented in Table 5.

Table 5. The rankings of supplier's by G-numbers, Importance and necessity concepts.

\begin{tabular}{llll}
\hline & Rank G-Numbers & Rank Importance component & Rank Necessity component \\
\hline $\mathrm{A}_{1}$ & 5 & 3 & 3 \\
$\mathrm{~A}_{2}$ & 6 & 6 & 2 \\
$\mathrm{~A}_{3}$ & 3 & 2 & 5 \\
$\mathrm{~A}_{4}$ & 2 & 5 & 1 \\
$\mathrm{~A}_{5}$ & 4 & 4 & 4 \\
$\mathrm{~A}_{6}$ & 1 & 1 & 6 \\
\hline
\end{tabular}


According to the results of this case, If only the Importance component is considered for the ranking of suppliers, the ranking will be $\left(\mathrm{A}_{6}>\mathrm{A}_{3}>\mathrm{A}_{1}>\mathrm{A}_{5}>\mathrm{A}_{4}>\mathrm{A}_{2}\right)$. And by considering the necessity component alone, the ranking will be $\left(A_{4}>A_{2}>A_{1}>A_{5}>A_{3}>A_{6}\right)$. By integrating the Importance and Necessity concepts in the form of $\mathrm{G}$ Numbers, the final ranking will be $\left(A_{6}>A_{4}>A_{3}>A_{5}>A_{1}>A_{2}\right)$. The results show that consideration of both components of necessity and importance, shows different rankings.

By comparing Rank of Importance component and GNumbers, the first, third and fourth suppliers, with importance component, were ranked in priority third, second and fifth respectively while, in the G-Numbers ranking, the suppliers were ranked fifth, third and second and the rest of suppliers, were placed in the same ranks. It should be admitted, that ranking by necessity concept base, can be different by Importance concept (Like this example). This reflects the difference in the nature of necessity and importance concepts. Therefore, the decision maker can consider different weights $(\alpha, \beta)$ for each of these (Importance-Necessity) concepts. Ranking of supplier selection example, for different weights of $\alpha$ and $\beta$ is presented in table 6 .

Table 6. Ranking of suppliers with different weights of $\alpha$ and $\beta$.

\begin{tabular}{llllllll}
\hline $\boldsymbol{\alpha}$ & $\boldsymbol{\beta}$ & $\mathbf{A}_{\mathbf{1}}$ & $\mathbf{A}_{\mathbf{2}}$ & $\mathbf{A}_{\mathbf{3}}$ & $\mathbf{A}_{\mathbf{4}}$ & $\mathbf{A}_{\mathbf{5}}$ & $\mathbf{A}_{\mathbf{6}}$ \\
\hline 1 & 0 & & 6 & 2 & 5 & 4 & 1 \\
0.9 & 0.1 & 3 & 6 & 2 & 5 & 4 & 1 \\
0.8 & 0.2 & 5 & 6 & 2 & 3 & 4 & 1 \\
0.7 & 0.3 & 5 & 6 & 3 & 2 & 4 & 1 \\
0.6 & 0.4 & 5 & 6 & 3 & 1 & 4 & 2 \\
0.5 & 0.5 & 5 & 6 & 2 & 1 & 4 & 3 \\
0.4 & 0.6 & 5 & 2 & 3 & 1 & 4 & 6 \\
0.3 & 0.7 & 3 & 2 & 5 & 1 & 4 & 6 \\
0.2 & 0.8 & 3 & 2 & 5 & 1 & 4 & 6 \\
0.1 & 0.9 & 3 & 2 & 5 & 1 & 4 & 6 \\
0 & 1 & 3 & 2 & 5 & 1 & 4 & 6 \\
\hline
\end{tabular}

\section{Conclusions}

Exact information is vital in planning, decision-making, and information management processes. In this study, a new concept called G-numbers is proposed to represent uncertain information. The concept of G-numbers, consider not only the concept of importance but also that of necessity in decision-making process. This new concept can be a step towards augmenting the human ability to make logical decisions in an environment of uncertainty and imprecision. The present study offers the arithmetic operations with Gnumbers and presents a numerical example to demonstrate the efficiency of the proposed approach. This approach can be used in many areas, including decision making, risk assessment, and information management. The proposed approach can be applied to some real-world issues and its results can be compared with those yielded from other approaches to improve the validation of the respective approach and investigate its applicability and usefulness. This approach can also be extended by applying it to group decision making.

\section{References}

[1] Zadeh, L. A., Fuzzy sets. Information and control, 1965. 8 (3): p. 338-353.

[2] Zadeh, L. A., A note on Z-numbers. Information Sciences, 2011. 181 (14): p. 2923-2932.

[3] Deng, Y., D numbers: theory and applications. Journal of Information \& Computational Science, 2012. 9 (9): p. 24212428 .

[4] Seiti, H., A. Hafezalkotob, and L. Martínez, R-numbers, a new risk modeling associated with fuzzy numbers and its application to decision making. Information Sciences, 2019. 483: p. 206-231.

[5] Memari, A., et al., Sustainable supplier selection: A multicriteria intuitionistic fuzzy TOPSIS method. Journal of Manufacturing Systems, 2019. 50: p. 9-24.

[6] Aboutorab, H., et al., ZBWM: The Z-number extension of Best Worst Method and its application for supplier development. Expert Systems with Applications, 2018. 107: p. 115-125.

[7] Mohammadi, A. and S. A. Darestani, Green supplier selection problem using TOPSIS extended by $D$ numbers in tractor manufacturing industry. International Journal of Services and Operations Management, 2019. 32 (3): p. 327-338.

[8] Aqlan, F. and S. S. Lam, A fuzzy-based integrated framework for supply chain risk assessment. International Journal of Production Economics, 2015. 161: p. 54-63.

[9] Abiyev, R. H., et al., Estimation of Food Security Risk Level Using Z-Number-Based Fuzzy System. Journal of Food Quality, 2018. 2018.

[10] Bian, T., et al., Failure mode and effects analysis based on D numbers and TOPSIS. Quality and Reliability Engineering International, 2018. 34 (4): p. 501-515.

[11] Capuano, N., et al., Fuzzy group decision making with incomplete information guided by social influence. IEEE Transactions on Fuzzy Systems, 2018. 26 (3): p. 1704-1718.

[12] Deng, X. and Y. Deng, D-AHP method with different credibility of information. Soft Computing, 2019. 23 (2): p. 683-691.

[13] Baer, D., Dwight Eisenhower Nailed A Major Insight About Productivity. Business Insider, 2014.

[14] Eisenhower, D., Address at the Second Assembly of the World Council of Churches, Evanston, Illinois. August, 1954. 19: p. 1954.

[15] Zavadskas, E.K., Z. Turskis, and S. Kildiene, State of art surveys of overviews on MCDM/MADM methods. Technological and economic development of economy, 2014. 20 (1): p. 165-179.

[16] Jafarzadeh Ghoushchi, S., Yousefi, S., \& Khazaeili, M., An extended FMEA approach based on the Z-MOORA and fuzzy $B W M$ for prioritization of failures. Applied Soft Computing, 2019. DOI: $10.1016 /$ j.asoc.2019.105505. 
[17] Gupta, M. M., Introduction to fuzzy arithmetic: Theory and applications. 1985: New York, NY: Van Nostrand Reinhold Company.
[18] Chen, C.-T., Extensions of the TOPSIS for group decisionmaking under fuzzy environment. Fuzzy sets and systems, 2000. 114 (1): p. 1-9. 